\title{
The complete genome sequence of the meat-borne lactic acid bacterium Lactobacillus sakei 23K
}

\author{
Stéphane Chaillou ${ }^{1}$, Marie-Christine Champomier-Vergès ${ }^{1}$, Monique Cornet ${ }^{1}$, Anne-Marie Crutz-Le Coq ${ }^{1}$, \\ Anne-Marie Dudez ${ }^{1}$, Véronique Martin ${ }^{1}$, Sophie Beaufils ${ }^{1}$, Emmanuelle Darbon-Rongère ${ }^{1}$, Robert Bossy ${ }^{2}$, \\ Valentin Loux $^{2} \&$ Monique Zagorec ${ }^{1}$ \\ Lactobacillus sakei is a psychrotrophic lactic acid bacterium found naturally on fresh meat and fish. This microorganism is \\ widely used in the manufacture of fermented meats and has biotechnological potential in biopreservation and food safety. We \\ have explored the 1,884,661-base-pair (bp) circular chromosome of strain $23 \mathrm{~K}$ encoding 1,883 predicted genes. Genome \\ sequencing revealed a specialized metabolic repertoire, including purine nucleoside scavenging that may contribute to an \\ ability to successfully compete on raw meat products. Many genes appear responsible for robustness during the rigors of food \\ processing - particularly resilience against changing redox and oxygen levels. Genes potentially responsible for biofilm formation \\ and cellular aggregation that may assist the organism to colonize meat surfaces were also identified. This genome project is an \\ initial step for investigating new biotechnological approaches to meat and fish processing and for exploring fundamental aspects \\ of bacterial adaptation to these specific environments.
}

Fresh meat and fish are nutritious but highly perishable foods. During production and storage they are exposed to unavoidable microbial contamination from the processing environment. Such contamination may include spoilage organisms and pathogens. It is therefore a priority for food processors to restrict the growth of contaminants so that they do not develop to potentially dangerous levels. Several methods are used, including vacuum-packaging, chemical preservation and chilling in addition to the more traditional practices of fermentation, salting and smoking. In recent years, however, highly processed foods have fallen out of favor so the food industry has had to start exploring more 'natural' methods of processing ${ }^{1}$. The use of safe bacteria to curb growth of spoilage and disease-causing microorganisms (termed 'biopreservatives') is one such alternative, and the meat-borne lactic acid bacterium $L$. sakei shows excellent promise ${ }^{2-4}$. This food-associated organism and transient inhabitant of the human gut $^{5,6}$ has the ability to survive and grow on fresh meat, forming the dominant population when selective techniques are applied ${ }^{7,8}$. Some strains are widely used in Europe for artisanal and larger scale manufacture of fermented sausages because of their useful preservative properties. These include the ability to produce lactic acid, hydrogen peroxide and bacteriocins. However, it is generally accepted that a successful biopreservative for fresh meat must also be able to compete for space and nutrients with the myriad other bacteria normally found on raw products, and their development should also be controlled to avoid overgrowth and fermentation ${ }^{2,9}$. Before simple pilot-scale studies can be scaled up into useful commercial biopreservative applications, an understanding of these interactions between competing species is needed.
In this paper, we report the complete genome sequence of $L$. sakei strain $23 \mathrm{~K}$, which was originally isolated from a French sausage ${ }^{8}$, with particular reference to survival aspects and competition with other meat-borne bacteria.

\section{RESULTS \\ General genome features and comparative genomics}

The principal features of the L. sakei $23 \mathrm{~K}$ chromosome are shown in Figure $\mathbf{1}$ and Table 1 . The genome is predicted to contain 1,883 protein/peptide-encoding genes (Supplementary Table 1 online). We identified one prophage remnant and 12 complete insertion sequences (IS) mostly localized in two diametrically opposed A+T-rich regions, suggesting hotspots for genome evolution. Despite the modest size of the genome, it contains seven rRNA gene clusters; such a redundancy may contribute to the organism's ability to flourish in complex microbial ecosystems ${ }^{10}$. With regard to gene products, the L. sakei genome shares the highest level of conservation (66\%) with Lactobacillus plantarum, a versatile bacterium sometimes used as a starter in fermented meat products ${ }^{7}$. The level of conservation was lower with the intestinal lactobacilli: Lactobacillus johnsonii (42\%) and Lactobacillus acidophilus (43\%). A comparative analysis between the four sequenced lactobacilli is given in Supplementary Figure 1 online and Table 2. These analyses revealed that part of the $L$. sakei genetic repertoire absent from other lactobacilli was typical of that used by ubiquitous aerobic Gram-positive bacteria for oxidative stress response, or energy production pathways not normally associated with a lactic acid bacterium, and suggests biological functions that could have a role in adaptation to meat.

${ }^{1}$ Unité Flore Lactique et Environnement Carné. INRA Domaine de Vilvert, 78350 Jouy-en-Josas, France. ${ }^{2}$ Mathématique, Informatique et Génome. INRA Domaine de Vilvert, 78350 Jouy-en-Josas, France. Correspondence should be addressed to M.Z. (monique.zagorec@jouy.inra.fr). 


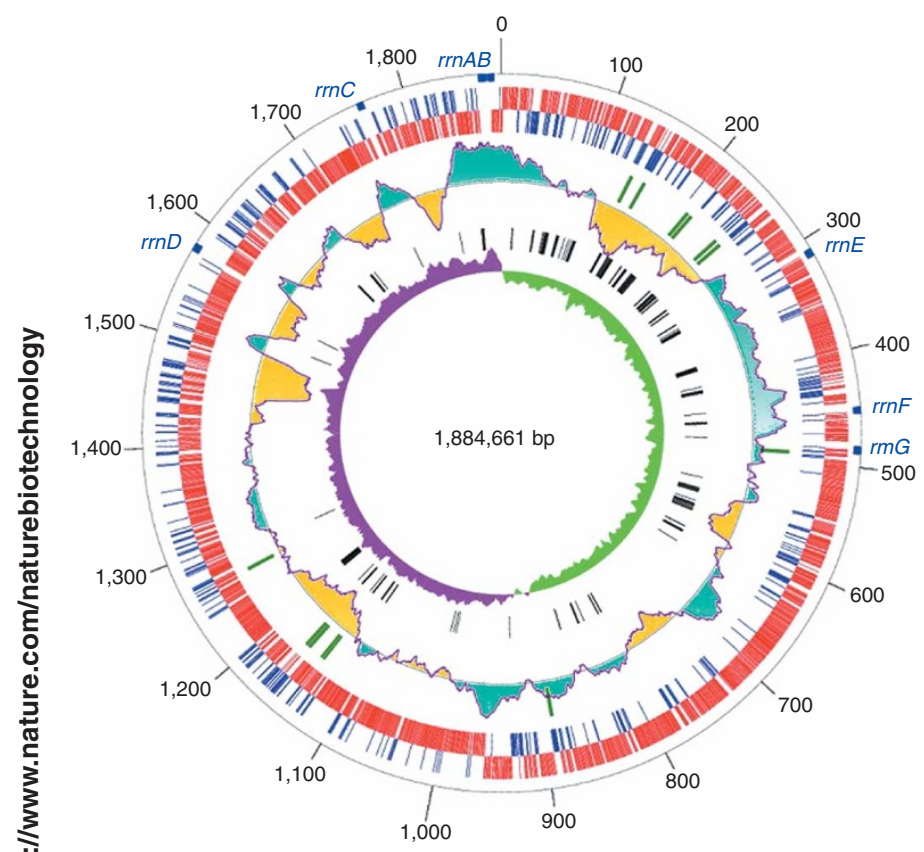

General metabolism and meat adaptation

Meat is a protein-rich subtrate and during its aging, proteolytic enzymes are released from lysosomes and amino acids are liberated from meat proteins without bacterial activity. Consistent with life in such an environment, L. sakei is auxotrophic for all amino acids, except aspartic and glutamic acids ${ }^{8}$. Genome analyses indicate that these two amino acids could potentially be produced by deamination of asparagine and glutamine respectively. We also noted an apparent absence of gene products relating to proteolysis such as proteinase PrtP, suggesting that L. sakei obtains nitrogen sources using only peptide and amino acid transporters.

Few transport systems are present for sugar uptake-a perhaps unsurprising finding considering the low diversity of carbohydrates available on meat. Only six phosphotransferase systems are present.
Figure 1 Map of the $L$. sakei $23 \mathrm{~K}$ chromosome. The outer circle represents the scale in $\mathrm{kb}$. Moving inward, the seven rRNA clusters are indicated in blue boxes along the outer circle. Protein coding sequences are shown in red (leading replication strand) or blue (lagging replication strand). The underlying irregular line (purple) and colored areas (orange/cyan) represents local $\mathrm{G}+\mathrm{C}$ content about the average (black circle). The average $\mathrm{G}+\mathrm{C}$ content is $41.25 \%$ and appears to be uneven along the chromosome, revealing regional islands. The window size was 50,000 nucleotides. IS elements are shown as thick green bars. The inner black bars indicate areas containing high concentrations of genes identified as important for meat adaptationespecially predominant in the first one-third of one chromosome's replichore. The inner purple and green circle represents GC-skew values.

They are transporters of glucose/mannose, N-acetylglucosamine, fructose, sucrose, trehalose and cellobiose. Catabolic pathways exist for five additional sugars (melibiose, gluconate, galactose, arabinose, ribose) and three carbohydrates (glycerol, malate and citrate).

L. sakei, like L. plantarum, is capable of synthesizing purines and pyrimidines de novo. Interestingly, the L. sakei genome contains a multiplicity of catabolic genes involved in exogenous nucleoside salvage pathways (Supplementary Table 2 online), such as those observed in Escherichia coli ${ }^{11}$ and Bacillus subtilis ${ }^{12}$. Indeed, growth experiments showed that $L$. sakei catabolizes inosine and adenosine for energy production (Supplementary Fig. 2 online). Glucose is the preferred carbon source for $L$. sakei but this compound is rapidly exhausted in meat $^{13}$, so nucleoside metabolism might improve its survival on meat (Fig. 2). This may also explain the presence of a gene encoding methylglyoxal synthase (MgsA, LSA1157) in the L. sakei genome and so far, this enzyme is a unique feature among lactic acid bacteria. In E. coli, methylglyoxal bypass of glycolysis occurs as a response to phosphate starvation or uncontrolled carbohydrate metabolism ${ }^{14}$. Such flexibility in L. sakei glycolytic processes may therefore reflect a requirement to deal with frequent glucose starvation and to modulate carbon flux during co-metabolism of alternative carbon sources. As with other Firmicutes, the detoxification route of methylglyoxal remains unknown in L. sakei. However, one possibility is suggested by a putative oxidoreductase encoded by LSA1158 contiguous with $m g s A$, which might reduce methylglyoxal to lactaldehyde. This

Table 1 Chromosome features of sequenced lactobacilli

\begin{tabular}{|c|c|c|c|c|c|}
\hline & & L. sakei $23 \mathrm{~K}$ & L. plantarum WCFS1 ${ }^{\mathrm{a}}$ & L. johnsonii NCC533a & L. acidophilus NCFMa \\
\hline Size (bp) & & $1,884,661$ & $3,308,274$ & $1,992,676$ & $1,993,564$ \\
\hline GC content (\%) & & 41.25 & 45.60 & 34.60 & 34.71 \\
\hline Coding density & & $86.9 \%$ & $84.1 \%$ & $89.3 \%$ & $87.9 \%$ \\
\hline \multirow[t]{6}{*}{ ORFs } & Protein-coding genes (CDSs) & 1,883 & 3,009 & 1,821 & 1,864 \\
\hline & Gene density (gene per kb) & 0.995 & 0.909 & 0.913 & 0.935 \\
\hline & Average gene length (bases per gene) & 885 & 920 & 977 & 943 \\
\hline & Genes on the leading replication strand (\%) & $78 \%$ & $74 \%$ & $73 \%$ & $77 \%$ \\
\hline & Pseudogenes (associated CDSs) ${ }^{b}$ & $30(61)$ & NA & NA & NA \\
\hline & CDSs with no database match ${ }^{c}$ & $254(13.5 \%)$ & $293(9.7 \%)$ & $150(8.2 \%)$ & $186(10 \%)$ \\
\hline \multirow[t]{2}{*}{ RNAs } & rRNAs (16S-23S-5S) & 7 & 5 & 4 & 4 \\
\hline & tRNAs & 63 (40 species) & 62 & 79 & 61 \\
\hline \multirow[t]{3}{*}{ Mobile elements } & IS elements ${ }^{d}$ & 12 & 15 & 14 & 17 \\
\hline & Prophages & 0 & 2 & 2 & 0 \\
\hline & Prophages remnant & 1 & 2 & 1 & 3 \\
\hline
\end{tabular}

${ }^{a}$ Data are derived from original annotation ${ }^{31,32,37}$. ${ }^{b} \mathrm{NA}$, not available in original annotation. ${ }^{\mathrm{C}}$ No significant similarity to any other protein in database. BLAST searches for the three lactobacilli previously published were updated. ${ }^{\text {dThe }} 12$ IS elements of $L$. sakei $23 \mathrm{~K}$ are composed of IS 1520 (IS3 family) ${ }^{50}$, five copies; IS $L s a 1$ (IS30 family), three copies; IS $L s a 2$ (IS150 family), three copies and ISLsa3 (IS4 family), one copy. A very limited degree of genome synteny was found between $L$. sakei and the three other species (data not shown). 
compound is possibly further reduced to L-lactate by the ironcontaining aldehyde dehydrogenase LSA0379.

When sugar diffusion from deeper meat layers declines and cannot replenish the supply for microbial growth on the meat surface, free amino acids are utilized ${ }^{13}$. The effective survival of $L$. sakei on meat products presumably involves catabolism of arginine, which is abundant in meat. The arginine deiminase (ADI) pathway was shown to enhance survival of $L$. sakei under anaerobiosis and also during stationary phase ${ }^{15,16}$. The importance of arginine catabolism is further supported by the identification of a second putative ADI pathway (Fig. 2) containing two putative peptidyl-arginine deaminases having $50 \%$ identity with that of Porphyromonas gingivalis ${ }^{17}$. These enzymes may contribute to the ADI pathway by catabolizing peptide-linked arginine not yet released as free amino acid, and could help L. sakei to scavenge arginine faster than competitors. It should be noted at this point that genes encoding tyrosine and histidine catabolic activities are absent in the $L$. sakei genome. From a biopreservation perspective this is important because, unlike many meat-borne microorganisms ${ }^{18}$, L. sakei does not produce tyramine and histamine, which are biogenic amines with deleterious effects on human health ${ }^{19}$.

\section{Psychotrophic character and salt tolerance}

L. sakei is capable of growing on meat during refrigeration and in the presence of curing salts $(3-9 \% \mathrm{NaCl})$. These two processes are widely used, sometimes in combination, to achieve a desired storage life. A key factor in L. sakei's acclimation to cold and salt could be its ability to efficiently accumulate osmo- and cryoprotective solutes such as betaine and carnitine. It is likely that accumulation of these compounds is driven by three ABC uptake systems (LSA0616 to LSA0619, LSA1694 to LSA1696, LSA1869-LSA1870), together with a $\mathrm{Na}^{+}-$ dependent symporter (LSA0264). This genetic repertoire is similar to that found in Listeria monocytogenes, an organism also known for salt and cold resistance ${ }^{20,21}$. Similarly, L. sakei has more putative cold stress proteins (LSA0768, LSA0946, LSA1152, LSA1561) than other lactobacilli, but as many as L. monocytogenes. Under high salt conditions, $L$. sakei probably uses extracellular sodium to drive substrate cotransport or export, since we found at least six $\mathrm{Na}^{+} / \mathrm{H}^{+}$antiporters

Table 2 Comparative functional distribution in sequenced lactobacilli

\begin{tabular}{|c|c|c|c|c|}
\hline & L. sakei $23 \mathrm{~K}$ & L. plantarum WCFS1 ${ }^{a}$ & L. johnsonii NCC533 ${ }^{a}$ & L. acidophilus NCFM ${ }^{a}$ \\
\hline \multicolumn{5}{|l|}{ Energy production pathways } \\
\hline Sugar-transporting phosphotransferase systems & 6 & 25 & 16 & 20 \\
\hline Pyruvate-dissimilatory pathways ${ }^{b}$ & Versatile & Versatile & Limited & Limited \\
\hline Glycolytic methylglyoxal bypass ${ }^{\mathrm{C}}$ & Possible & Absent & Absent & Absent \\
\hline Amino acid catabolism ${ }^{c}$ & Arginine, aspartate, threonine & None identified & Ornithine & Ornithine \\
\hline Nucleoside/nucleotide transporters & 10 & 6 & 3 & 5 \\
\hline Nucleoside putative catabolic enzymes ${ }^{c}$ & $\begin{array}{c}3 \text { phosphorylases, } 4 \text { deaminases } \\
1 \text { phosphopentomutase }\end{array}$ & 2 deaminases & 1 deaminase & 1 deaminase \\
\hline \multicolumn{5}{|l|}{ Nitrogen metabolism } \\
\hline Extracellular proteinase & None identified & None identified & PrtP & PrtP \\
\hline Amino acid/peptide transporters; peptidases & $24-22$ & $22-19$ & $25-25$ & $24-20$ \\
\hline Amino acid biosynthesis & Asparagine, glutamine & All but branched chain & Glycine, asparagine, aspartate & $\sim 10$ amino acids \\
\hline Purines \& pyrimidines de novo synthesis & Purines, pyrimidines & Purines, pyrimidines & Pyrimidines & Pyrimidines \\
\hline \multicolumn{5}{|l|}{ Redox \& iron metabolism ${ }^{c}$} \\
\hline Peroxides metabolism & 8 & 13 & 3 & 3 \\
\hline Thioredoxin \& thioredoxin reductases & $4-3$ & $4-2$ & $2-1$ & $4-3$ \\
\hline Uncharacterized oxidoreductases & 40 & 41 & 14 & 19 \\
\hline Iron-loaded siderophore transporters & 1 & 2 & None identified & None identified \\
\hline Ferric uptake regulators & 3 & 2 & None identified & None identified \\
\hline \multicolumn{5}{|l|}{ Cryo- and osmotolerance ${ }^{c}$} \\
\hline Compatible solute transporters & 4 & 3 & 1 & 1 \\
\hline Cold shock proteins (CspA family) & 4 & 3 & 1 & 1 \\
\hline \multicolumn{5}{|l|}{ Detoxification } \\
\hline Bile-salt hydrolases & 1 & 4 & 3 & 2 \\
\hline Aromatic hydrocarbon decarboxylases & 3 & 2 & 1 & 1 \\
\hline \multicolumn{5}{|l|}{ Cell-surface component ${ }^{d}$} \\
\hline Proteins with an LPXTG anchor motif & 4 & 25 & 14 & 6 \\
\hline Putative mucin-binding proteins (MUB) & 0 & 2 & 4 & 5 \\
\hline Proteins with an WXL C-terminal domain & 15 & 19 & None identified & None identified \\
\hline Two-component systems & 10 & 13 & 9 & 9 \\
\hline
\end{tabular}

${ }^{a}$ Data are derived from original annotation ${ }^{31,32,37}$ or from comparative analysis of Clusters of Orthologous Groups. The values indicate the number of genes or gene clusters identified for the corresponding metabolism. bersatile indicates a mixed-acid fermentation capacity to produce lactate, acetate, formate, ethanol, acetoin and 2,3 butanediol. Limited indicates a homolactic fermentation capacity only (exclusively lactate production). ${ }^{C}$ These metabolisms are shared with other ubiquitous Gram-positive bacteria. ${ }^{d}$ The proteins were searched using corresponding motifs or protein domains. 
Figure 2 L. sakei $23 \mathrm{~K}$ energy production pathways contributing to meat adaptation. Meat and fish products typically contain low levels of sugars. Glycogen is the main source of glucose whereas ribose is released by ATP hydrolysis ${ }^{13}$ (denoted in blue outside the cell). Inosine and IMP are the most abundant intermediates of ATP breakdown with meat concentrations reaching as much as twice that of glucose ${ }^{13}$. Enzyme occurrence in sequenced lactobacilli is shown with color code: gray (all), blue ( $L$. sakei, L. plantarum), green ( $L$. sakei and ubiquitous Gram-positive bacteria). Enzymes involved in nucleoside scavenging include (1) one adenosine deaminase (LSA0086), (2) three inosine-preferring hydrolases (LSA0252,

के LSA0533, LSA830) involved in the release of the ribose moiety and (3) three nucleoside phosphorylases (LSA0776, LSA0797, LSA0801) producing ribose 1-phosphate - those enzymatic activities composing the major pathway for nucleoside scavenging in $E$. coli and B. subtilis ${ }^{11,12}$ Subsequent metabolism of ribose-derived molecules for energy production include (4) phosphopentomutase (LSA0796), (5) ribokinases (LSA0202, LSA0254) and (6) ribose 5-P isomerase (LSA1685). The elements of the L. sakei glycolytic pathway that may facilitate energy production regulation (during glucose starvation or growth at low temperatures) are highlighed in green. This includes (7) fructose-1,6-bisphosphatase (LSA1778); (8) pyruvate phosphodikinase (LSA1141) involved in the anaplerotic conversion of pyruvate to PEP (phosphoenolpyruvate) and (9) a possible methylglyoxal bypass with methylglyoxal synthase (LSA1157), which may subsequently be reduced to (10) lactaldehyde (LSA1158) and to (11) lactate (LSA0379). The four steps from GA-3P to PEP are shown as one. The duplicated ADI pathways involved in catabolism of the secondary energy source arginine are shown in pink arrows: (12) arginine (LSA0370) and peptidyl-arginine (LSA0070, LSA0072) deaminases, (13) ornithine transcarbamoylases

윽 (LSA0067, LSA0371) and (14) carbamate kinases (LSA0071, LSA0372)

$[2 \mathrm{H}], \mathrm{NADH}$ reducing equivalent; $\mathrm{P}_{\mathrm{i}}$, phosphate; G6P, glucose 6-P; F6P,

fructose 6-P; FDP, fructose 1,6-diphosphate; GA-3P, glyceradehyde 3-

O phosphate; DHAP, dihydroxyacetone phosphate; PTS, phosphotransferase system; IMP, inosine 5-P; AMP, adenosine 5-P.

and one ATP-dependent $\mathrm{Na}^{+}$efflux pump (LSA0051, LSA0052) that could modulate and regulate the sodium-motive force. Regarding its resilience to cold, one study suggested that $L$. sakei may use a combination of mechanisms ${ }^{22}$, including a modification of carbohydrate metabolism (downregulation of glycolysis) and stimulation of oxidative stress.

\section{Redox and antioxidant activities}

Meat processing usually generates changes in product oxygen levels and redox potential. For example, oxygen levels are reduced in processes such as vacuum-packaging, fermentation or when a reducing agent such as nitrite salt is used. Conversely, oxygen levels may increase when processing occurs in the presence of air and during storage when air gains access to the meat surface over time. Analysis of Lactobacillus genomes for redox activities revealed remarkable differences among species (Supplementary Table 3 online). In comparison to intestinal lactobacilli, both $L$. sakei and L. plantarum are surprisingly well equipped to cope with changing oxygen conditions. But L. sakei appears to deal more effectively with some toxic oxygen byproducts, such as superoxide $\left(\mathrm{O}_{2}^{-}\right)$and organic hydroperoxide (Fig. 3). At least two enzymes, NADH oxidase (Nox, LSA0802) and manganese-dependent superoxide dismutase (SodA, LSA0896), could catalyze the production of $\mathrm{H}_{2} \mathrm{O}_{2}$ from $\mathrm{O}_{2}^{-}$, possibly an important issue in biopreservation since $\mathrm{H}_{2} \mathrm{O}_{2}$-generating strains are suspected to exert an antibacterial effect against contaminant organisms in foods ${ }^{23}$. Reduction of peroxide is achieved by thiol peroxidase (Tpx, LSA0104), NADH peroxidase (Npr, LSA0575), an iron-containing Dye-type peroxidase (LSA1831), a heme-dependent catalase (KatA, LSA0171) ${ }^{24}$ and finally one organic hydroperoxide-resistance protein (OhrA, LSA0552). L. sakei shares with most lactobacilli several reducing mechanisms that restore protein activity after oxidation

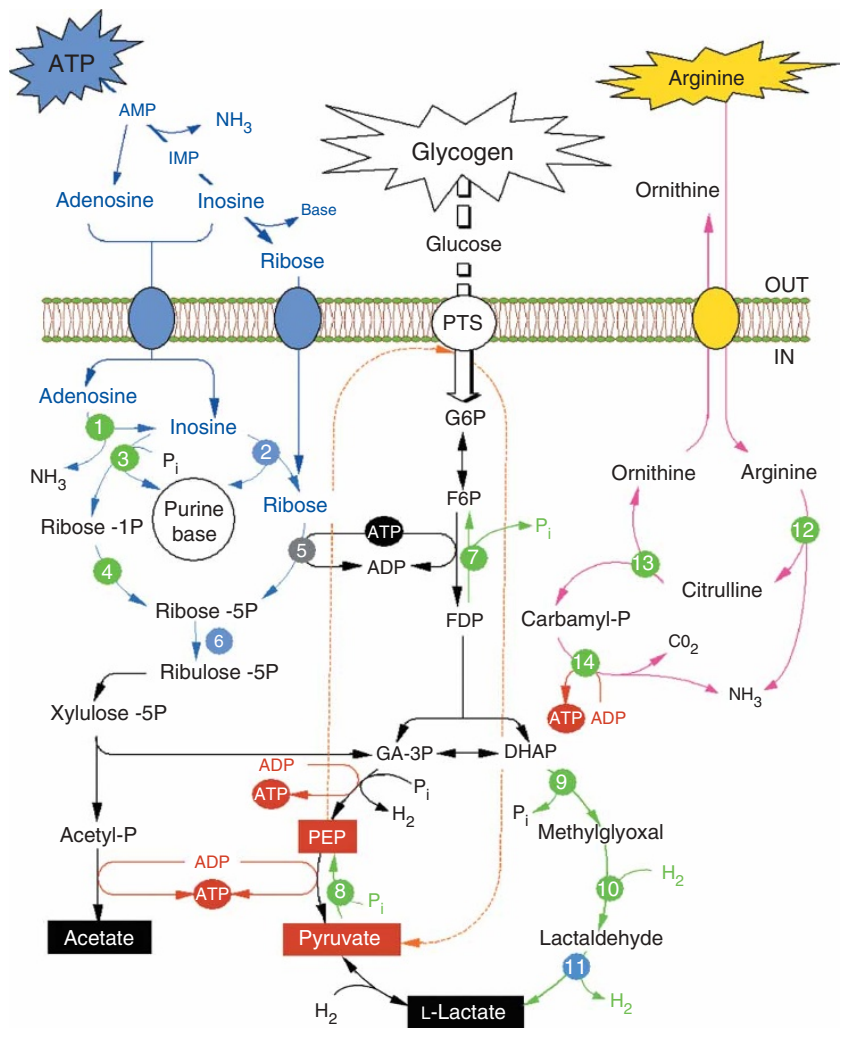

damage. These include two peptide methionine sulfoxide reductases (LSA0865, LSA0866), which counter methionine oxidation, and a redundancy of thioredoxin (LSA0218, LSA0307, LSA0394, LSA0634)/ thioredoxin reductase (LSA0403, LSA0435, LSA0520) systems. L. sakei possesses an additional glutathione/glutaredoxin (LSA0942)/ glutathione reductase (LSA1871) system, in which glutathione is presumably synthesized by the multidomain GshF protein ${ }^{25}$ (LSA0840). Such a system seems to also be present in L. plantarum. Presumably, each system has its own redox characteristic and fulfills different functional roles to control the intracellular redox balance. For example, thioredoxin (LSA0218) is located within a cluster comprising a putative thiosulfate sulfurtransferase (LSA0217) and a putative cyanate transporter (LSA0219_b). It is probable that LSA218 participates in detoxifying cyanate to thiocyanate. Once formed, thiocyanate, in the presence of $\mathrm{H}_{2} \mathrm{O}_{2}$, might react with peroxidases from the growth environment to form hypothiocyanate ${ }^{23}$, an ion with well-known bacteriostatic effects against Gram-negative bacteria ${ }^{26}$, which would contribute to the antibacterial activity of $L$. sakei in meat. A large arsenal of putative oxidoreductases with few homologs among lactobacilli have also been identified (Supplementary Table 3 online) suggesting an efficient capacity for maintaining redox balance through tight control of the $\mathrm{NADP} / \mathrm{NADPH}^{+}$ratio and the use of a wide range of electron acceptors.

\section{Heme and iron requirement}

Meat is an iron-rich substrate and reactive oxygen species can arise from the combination of oxygen and iron through Fenton reactions (Fig. 3). Although lactobacilli are not generally considered as requiring $^{27}$ iron, the heme-dependent catalase of L. sakei indicates that heme acquisition is important for the oxidative stress response of the organism. L. sakei cannot synthesize heme de novo and therefore 


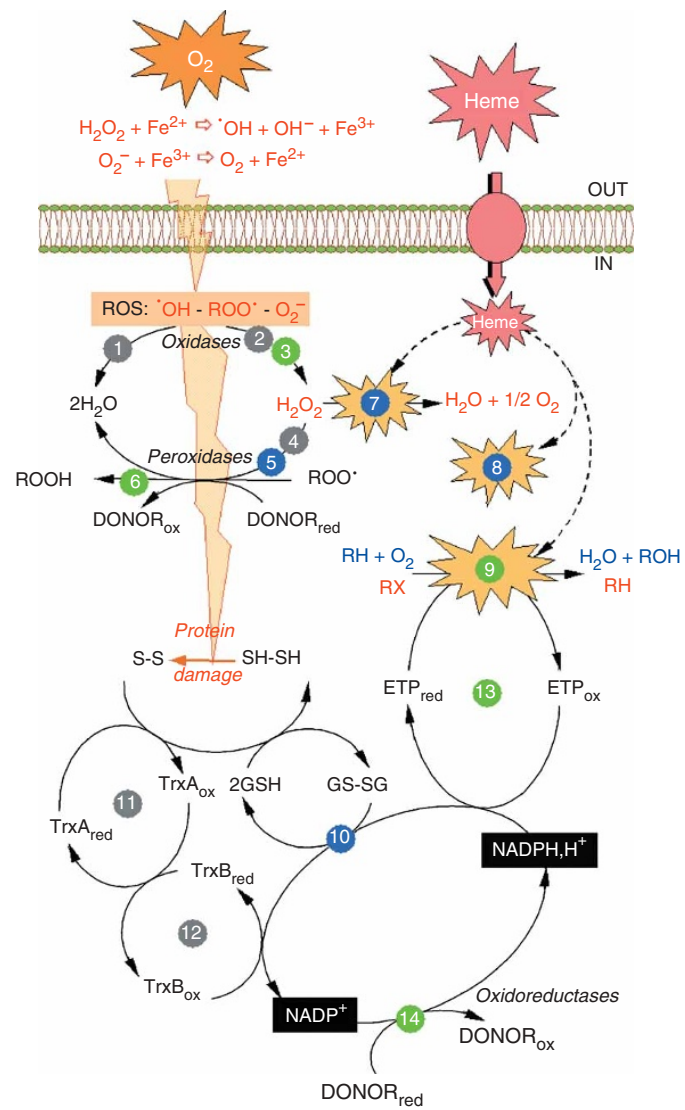

would take up heme from meat. At least four putative iron transport systems have been detected in L. sakei, LSA0246, LSA0282 to LSA0284, LSA1699, and LSA0399 to LSA0402. LSA0399-0402 may be transcriptionally coupled with a thioredoxin reductase (LSA0403) and is predicted to take up iron-loaded siderophores and possibly heme. Genome analysis has further revealed two putative cytochromes of unknown functions, giving additional support to the view that heme is used by L. sakei. The first one (LSA1311) belongs to the ubiquitous cytochrome b5 family (PF00173); the second one (LSA1182/1183) is a cytochrome P450 (transcriptionally interrupted by ISLsa3 in strain $23 \mathrm{~K}$ but intact in other strains; unpublished data), a protein putatively acting as monoxygenase or reductase when complexed with ferredoxin (LSA1022) and flavodoxin (LSA1516) ${ }^{28}$. Furthermore, three irondependent regulators belonging to the Fur family (LSA0109, LSA0466, LSA1029) were observed, as is the case for numerous Gram-positive pathogens, but not for other lactobacilli. Although the exact role of these regulators is unclear, they might be involved in resistance to oxidative stress or regulation of iron transport proteins ${ }^{29,30}$.

\section{Cell surface components for adhesion and cellular aggregation}

Little similarity was found between the predicted cell-surface components identified in $L$. sakei and those of other lactobacilli, as would reflect their different ecological niches. For instance, L. sakei lacks homologs of MUB proteins ${ }^{31,32}$ for adherence to intestinal mucus. On the other hand, L. sakei may colonize the meat surface through aggregation and biofilm formation with a set of components having no homolog in other lactobacilli. One candidate for biofilm formation is a large 1,987-residue protein (LSA0534) composed of 15 perfectly conserved 100-amino-acid repeats and of an LPXTG motif (PF00746) involved in covalent anchoring to the cell wall. It demonstrates 37\%
Figure 3 Versatile redox metabolism of $L$. sakei 23K. Reactive Oxygen Species (ROS) can be produced in meat by enzymatic or spontaneous processes such as Fenton reactions (indicated in red outside the cell). Resistance to hydrogen peroxide, redox changes due to food processing, and protein repair due to ROS are catalyzed by a set of redundant redox systems. Color code for enzyme occurrence is the same as for Figure 2. $(1,2) \mathrm{NADH}$ oxidases (LSA0802 for $\mathrm{H}_{2} \mathrm{O}_{2}$-forming, LSA0312 for waterforming); (3) superoxide dismutase (LSA0896); (4) thiol peroxidase (LSA0104); (5) NADH peroxidase (LSA0575), dye-type peroxidase (LSA1831); (6) organic hydroperoxide-resistance protein (LSA0552). Heme-dependent oxidoreductases are shown in star-boxes, the roles of the cytochromes are yet to be identified: (7) catalase (LSA0172); (8) putative cytochrome b5 (LSA1311); (9) cytochrome P450 (LSA1182/83, mutated in strain $23 \mathrm{~K}$ but functional in other strains) may function either as monoxygenase (blue reaction), or under anaerobiosis, as a reductase (red reaction). S-S, oxidated thiol groups; $\mathrm{SH}-\mathrm{SH}$, reduced thiol groups; GSH or GS-SG, oxidated or reduced glutathione; (10) glutaredoxin (LSA0942), glutathione reductase (LSA1871); (11) TrxA, thioredoxins; (12) TrxB, thioredoxin reductases; $X_{0 x}$ and $X_{\text {red }}$, oxidated or reduced state, respectively; (13) ETPs, electron transfert proteins (ferredoxin, LSA1022 and flavodoxin, LSA1516); (14) uncharacterized oxidoreductase.

similarity to Biofilm Associated Protein (BAP) found in bovine Staphylococcus aureus strains ${ }^{33}$. We further identified four paralogous proteins predicted to mediate autoaggregation and adherence to collagen $^{34}$. They have a central region rich in Ser/Thr/Gln, demonstrate physical properties quite similar to cell surface-layer proteins, and can be further divided into two sets. One set is composed of two proteins (LSA1159, LSA1165) containing an N-terminal cell-wall binding LysM domain (PF01476) and a C-terminal domain observed in autoaggregation-promoting factors of intestinal lactobacilli and enterococci ${ }^{35}$. The second set (LSA0194, LSA0313) are proteins containing a C-terminal cell-wall binding P60 domain (PF00877) and a $\mathrm{N}$-terminal sequence of two repeated GW-like modules involved in lipoteichoic acid binding ${ }^{36}$. Each set is composed of one slightly acidic protein (pI 5.3-6.4; LSA0313 and LSA1159) and one highly basic protein ( $\mathrm{pI}>8.9-8.7$, LSA0194 and LSA1165) suggesting different binding properties or environment-dependent production (meat surface versus intestinal tract).

We also identified several gene clusters potentially encoding a novel type of multicomponent complex on the surface of the bacterium. This includes fifteen proteins having a C-terminal WXL-like domain ${ }^{37}$ with a $\operatorname{YxxT}(\mathrm{L} / \mathrm{I}) \mathrm{TW}(\mathrm{T} / \mathrm{S}) \mathrm{L}$ motif, which was identified in Lactobacillus coryneformis $\mathrm{Cpf}$ surface protein mediating coaggregation with E. $\operatorname{coli}^{38}$. Within each cluster, the WXL proteins are present as pairs together with one or two genes encoding hydrophobic-tail proteins (Supplementary Fig. 3 online). These proteins have no homolog in databases and their precise role has yet to be determined. One WXLtype protein (LSA1731) may mediate adhesion to meat components for it has a central domain showing similarity to hemagglutinin of Staphylococcus epidermidis.

The genome of L. sakei also contains two distinct gene clusters (LSA1571 to LSA1585) and (LSA1510[a-g] to LSA1513) potentially involved in the production of surface polysaccharides, which could mediate attachment to the meat surface or the intestinal mucosa. The first cluster is predicted to be involved in synthesis of polysaccharidelinked teichoic acid because it contains homologs of both glycerol-3phosphate cytidyltransferases and glycosyl transferases. The second cluster, which encodes glycosyl transferases and one protein of unknown function (LSA1510_e) with a lipoprotein signal-peptidase domain (IPR001872), might be involved in a transfer activity of the polysaccharide to a surface component. 


\section{Transport and detoxification activities}

A total of 153 transport systems have been identified in L. sakei (Supplementary Table 4 online). Of these, 45 are possibly dedicated to multidrug resistance. With only one putative bile-salt hydrolase (LSA0210), L. sakei may be more sensitive to these toxic compounds than other lactobacilli. On the other hand, three enzymes may play a specific role in detoxification activities of aromatic compounds coming from exposure to spices and smoke, still used as traditional meat biopreservation methods ${ }^{26}$ : a $p$-coumaric acid decarboxylase (PdcA, LSA1701), and two putative aromatic hydrocarbon decarboxylases (LSA1190, LSA1776) of the MCD family (PF02627).

\section{Bacteriocin production and immunity}

L. sakei is well known for its ability to produce bacteriocins ${ }^{8}$, in particular the antilisterial peptide sakacin P. This property is often favored when selecting strains for biopreservation ${ }^{3,4}$. However, L. sakei strain $23 \mathrm{~K}$ is not a bacteriocin producer but has a rearranged chromosome-encoded sakacin P cluster (LSA0560_a to LSA569_b) containing regulatory function remnants and a mosaic of genes encoding putative bacteriocin-like peptides with cognate immunity proteins similar to those produced by L. plantarum ${ }^{39}$. This suggests that strain $23 \mathrm{~K}$ may possess an innate resistance towards bacteriocins produced by its closely related competitors. This resistance is probably as useful for competitive fitness as is a functional sakacin $\mathrm{P}$ due to the limited diffusion properties of bacteriocins in solid matrices ${ }^{2}$.

\section{DISCUSSION}

Analysis of the L. sakei genome reveals a combination of several strategies being used by the organism to adapt and grow on meat products. Important cellular functions relating to each strategy are encoded by a redundancy of genes likely to enhance the organism's robustness, and most probably help it to outgrow other competing bacteria. L. sakei is able to flexibly use meat components. Purine nucleosides, abundant in meat, are exploited for growth and energy production. This ability, unique among lactic acid bacteria, would facilitate $L$. sakei's growth on meat after glucose depletion. Subsequent (2) degradation of arginine has an important role for survival of L. sakei by avoiding cell death when carbon sources are lacking. L. sakei has an unusually flexible glycolytic pathway for a lactic acid bacterium, with a postulated methyglyoxal bypass that would adequately cater to its frequent need to modulate carbon fluxes, and perhaps to adapt to the types of cold stresses experienced in meat products.

L. sakei is a very robust microorganism that profits from a strong capacity to resist adverse environmental conditions encountered in processed food (antimicrobial substances, high salt concentration and changing redox conditions). The bacterium flourishes when oxygen is scarce, outperforming competition by aerobic bacteria. Despite a predilection for anaerobiosis, a versatile redox metabolism allows L. sakei to deal surprisingly well with oxidative stresses and facilitates its survival on fresh meat until better growth conditions are encountered. It seems that iron and heme acquisition is somehow correlated with such resistance, an ability characteristic of pathogenic bacteria.

Finally, biofilm formation and cellular (auto- or co-)aggregation appear to be predominant mechanisms that allow L. sakei to proliferate and colonize meat surfaces. This would be particularly important for overcoming nutrient fluctuations, and thereby for increasing the organism's capacity to compete ${ }^{40}$.

A detailed large-scale analysis of $L$. sakei metabolic pathways and their respective regulation during various growth conditions (e.g., different conditions of redox potential, refrigeration or addition of specific antimicrobial components) should provide new ideas for the design of efficient biopreservative cultures. Understanding L. sakei responses to oxidative stress is pivotal in devising other ways to preserve fresh meat because they have the potential to reduce bacterial contamination. The question can also be raised as to whether competitive iron/heme acquisition by $L$. sakei can be used to control pathogens in these products. Finally, these approaches could provide a better knowledge of the relationship between harmless and pathogenic bacteria in food matrices.

\section{METHODS}

Sequencing strategy. The L. sakei $23 \mathrm{~K}$ genome was determined using a conventional whole-shotgun strategy ${ }^{41}$. A clone library with an average insert size of $1-2 \mathrm{~kb}$ was constructed as described previously $\mathrm{y}^{42}$. Gap closure was carried out using a combination of PCR strategies (Vectorette PCR, Multiplex PCR) and genome mapping with closely related species followed by PCR amplification. Sequence mis-assemblies were checked against the $L$. sakei physical map ${ }^{42}$. All sequences $(\sim 15,500)$ were assembled in one contig using the Phred/Phrap/Consed software package ${ }^{43,44}$ generating a 4.5 -fold redundancy. Every consensus position had at least a double-clone coverage (sequenced from both strands) or sequences from three PCR products amplified from genomic DNA. The final mean error rate on nucleotide level was $<1$ per 50,000 bases.

Gene prediction and annotation. Genome annotation was performed using AGMIAL (http://genome.jouy.inra.fr/agmial)—a system using integrated gene prediction tools based on Hidden Markov Models in addition to protein and metabolic function predictive tools. Web-based software and databases were used to manually curate predicted genes after comparing data to public databases (COGs, Interpro, Pfam, Prints, Prodom, PROSITE, Smart, Swissprot and Tigrfams). Putative frameshifts were checked and corrected after resequencing with final frameshifts considered authentic. Genomic comparisons were carried out with $\mathrm{MuGen}^{45}$ and FindTarget ${ }^{46}$. Orthologous genes were analyzed using bidirectional BLAST ${ }^{47}$ best hit (E-value threshold of $10^{-10}$ ). Solute transporters were classified using the TC number system ${ }^{48}$.

Growth experiments. L. sakei $23 \mathrm{~K}$ was grown for $24 \mathrm{~h}$ at $30{ }^{\circ} \mathrm{C}$ in $\mathrm{MRS}^{49}$ broth prepared without glucose and supplemented with $25 \mathrm{mM}$ of specific sugars or nucleosides. Optical density was determined at $600 \mathrm{~nm}$.

Nucleotide sequence accession number. The sequence reported in this paper has been deposited in the EMBL database as Accession No. CR936503. The annotated genome sequence and the relevant AGMIAL database (Genome navigator, BLAST server and Metabolic comparison) are available at http://migale.jouy.inra.fr/sakei/.

Note: Supplementary information is available on the Nature Biotechnology website.

\section{ACKNOWLEDGMENTS}

We thank Rhys Jones and Alexandra Gruss for critical reading of the manuscript and Christophe Caron for his contribution on the Lactobacillus sakei genome server.

\section{COMPETING INTERESTS STATEMENT}

The authors declare that they have no competing financial interests.

Published online at http://www.nature.com/naturebiotechnology/

Reprints and permissions information is available online at http://npg.nature.com/ reprintsandpermissions/

1. Devlieghere, F., Vermeiren, L. \& Debevere, J. New preservation technologies: possibilities and limitations. Int. Dairy J. 14, 273-285 (2004).

2. Vermeiren, L., Devlieghere, F. \& Debevere, J. Evaluation of meat born lactic acid bacteria as protective cultures for the biopreservation of cooked meat products. Int. J. Food Microbiol. 96, 149-164 (2004).

3. Bredholt, S., Nesbakken, T. \& Holck, A. Industrial application of an antilisterial strain of Lactobacillus sakei as a protective culture and its effect on the sensory acceptability of cooked, sliced, vacuum-packaged meats. Int. J. Food Microbiol. 66, 191-196 (2001).

4. Vold, L., Holck, A., Wasteson, Y. \& Nissen, H. High levels background flora inhibits growth of Escherichia coli $0157 \mathrm{H7}$ in ground beef. Int. J. Food Microbiol. 56, 219-225 (2000). 
5. Walter, J. et al. Detection of Lactobacilli, Pediococcus, Leuconostoc, and Weisella species in Human feces by using group-specific PCR primers and denaturing gradient gel electrophoresis. Appl. Environ. Microbiol. 67, 2578-2585 (2001).

6. Dal Bello, F., Walter, J., Hammes, W.P. \& Hertel, C. Increased complexity of the species composition of lactic acid bacteria in Human feces revealed by alternative incubation condition. Microb. Ecol. 45, 455-463 (2003).

7. Hammes, W.P., Bantleon, A. \& Min, S. Lactic acid bacteria in meat fermentation. FEMS Microbiol. Rev. 87, 165-174 (1990).

8. Champomier-Vergès, M., Chaillou, S., Cornet, M. \& Zagorec, M. Lactobacillus sakei: recent developments and future prospects. Res. Micobiol. 153, 115-123 (2002).

9. Gram, L. \& Dalgaard, P. Fish spoilage bacteria - problems and solutions. Curr. Opin. Biotechnol. 13, 262-266 (2002)

10. Klappenbach, J.A., Dunbar, J.M. \& Schmidt, T.M. rRNA operon copy number reflects ecological strategies of bacteria. Appl. Environ. Microbiol. 66, 1328-1333 (2000).

11. Zalkin, H. \& Nygaard, P. in Escherichia coli and Salmonella typhimurium. Cellular and Molecular Biology edn. 2 (eds. Neidhardt F.C. et al.) 561-579, (ASM Press, Washington, DC, 1996).

12. Schuch, R., Garibian, A., Saxild, H.H., Piggot, P. \& Nygaard, P. Nucleosides as a carbon source in Bacillus subtilis: characterization of the drm-pupG operon. Microbiol. 145 2957-2966 (1999).

13. Gill, C.O. in Meat microbiology (ed. Brown, M.H.) 225-264 (Applied Science Publishers, New York, 1982)

14. Weber, J., Kayser, A. \& Rinas, U. Metabolic flux analysis of Escherichia coli in glucoselimited continuous culture. Dynamic response to famine and feast, activation of the methylglyoxal pathway and oscillatory behaviour. Microbiol. 151, 707-716 (2005).

15. Zúniga, M., Champomier-Vergès, M., Zagorec, M. \& Pérez-Martínez, G. Structural and functional analysis of the gene cluster encoding the enzymes of the arginine deiminase pathway of Lactobacillus sakei. J. Bacteriol. 180, 4154-4159 (1998).

16. Champomier-Vergès, $M$. et al. Relationships between arginine degradation, $\mathrm{pH}$ and survival in Lactobacillus sakei. FEMS Microbiol. Lett. 180, 297-304 (1999).

17. McGraw, W.T., Potempa, J., Farley, D. \& Travis, J. Purification, characterization and sequence analysis of a potential virulence factor from Porphyromonas gingivalis, peptitylarginine deiminase. Infect. Immun. 67, 3248-3256 (1999).

218. Masson, F., Talon, R. \& Montel, M.C. Histamine and tyramine production by bacteria from meat products. Int. J. Food Microbiol. 32, 199-207 (1996).

19. Bardócz, S. Polyamines in foods and their consequences for food quality and human health. Trends Food Sci. Technol. 6, 341-346 (1995).

20. Angelidis, A. \& Smith, G.M. Three transporters mediate uptake of glycine betaine and carnitine by Listeria monocytogenes in response to hyperosmotic stress. Appl. Environ. Microbiol. 69, 1013-1022 (2003).

21. Angelidis, A. \& Smith, G.M. Role of the glycine and carnitine transporters in adaptation of Listeria monocytogenes to chill stress in defined medium. Appl. Environ. Microbiol. 69, 7492-7498 (2003).

22. Marceau, A., Zagorec, M., Chaillou, S., Méra, T. \& Champomier-Vergès, M. Evidence for involvement of at least proteins in adaptation of Lactobacillus sakei to cold temperatures and addition of NaCl. Appl. Environ. Microbiol. 70, 7260-7268 (2004).

23. Condon, S. Response of lactic acid bacteria to oxygen. FEMS Microbiol. Rev. 46, 269-280 (1987).

24. Hertel, C., Schmidt, G., Fischer, M., Oellers, K. \& Hammes, W.P. Oxygen-dependent regulation of the expression of the catalase gene katA of Lactobacillus sakei LTH677. Appl. Environ. Microbiol. 64, 1359-1365 (1998).

50 25. Gopal, S. A multidomain fusion protein in Listeria monocytogenes catalyzes the two primary activities for glutathione biosynthesis. J. Bacteriol. 187, 3839-3847 (2005).

26. Davidson, P.M. in Food Microbiology, Fundamentals and Frontiers, edn. 1 (eds. Boyle, M.P., Beuchat, L.R. \& Montville, T.J.) 520-549 (ASM Press, Washington, DC, 1997).
27. Pandey, A., Bringel, F. \& Meyer, J.M. Iron requirement and search for siderophores in lactic acid bacteria. Appl. Microbiol. Biotechnol. 40, 735-739 (1994).

28. Mansuy, D. The great diversity of reactions catalyzed by cytochromes P450. Comp. Biochem. Physiol. C 121, 5-14 (1998).

29. Horsburgh, M.J., Clements, M.O., Crossley, H., Ingham, E. \& Foster, S.J. PerR controls oxidative stress resistance and iron storage proteins and is required for virulence in Staphylococcus aureus. Infect. Immun. 69, 3744-3754 (2001).

30. Rea, R.B., Gahan, C.G.M. \& Hill, C. Disruption of putative regulatory loci in Listeria monocytogenes demonstrates a significant role for Fur and PerR in virulence. Infect. Immun. 72, 717-727 (2004).

31. Pridmore, R.D. et al. The genome sequence of the probiotic intestinal bacterium Lactobacillus johnsonii NCC 533. Proc. Natl. Acad. Sci. USA 101, 2512-2517 (2004).

32. Altermann, E. et al. Complete genome sequence of the probiotic lactic acid bacterium Lactobacillus acidophilus NCFM. Proc. Natl. Acad. Sci. USA 102, 3906-3912 (2005).

33. Cucarella, C. et al. Bap, a Staphylococcus aureus surface protein involved in biofilm formation. J. Bacteriol. 183, 2888-2896 (2001).

34. Sillanpää, J. et al. Characterization of the collagen-binding S-layer protein CbsA of Lactobacillus crispatus. J. Bacteriol. 182, 6440-6450 (2000).

35. Ventura, M., Jankovic, I., Walker, D.C., Pridmore, R.D. \& Zink, R. Identification and characterization of novel proteins in Lactobacillus johnsonii and Lactobacillus gasseri. Appl. Environ. Microbiol. 68, 6172-6181 (2002).

36. Jonquières, R., Bierne, H., Fiedler, F., Gounon, P. \& Cossart, P. Interaction between the protein InIB of Listeria monocytogenes and lipoteichoic acid: a novel mechanism of protein association at the surface of Gram-positive bacteria. Mol. Microbiol. 34 902-914 (1999).

37. Kleerebezem, M. et al. Complete genome sequence of Lactobacillus plantarum WCFS1. Proc. Natl. Acad. Sci. USA 100, 1900-1995 (2004).

38. Schachtsiek, M., Hammes, W.P. \& Hertel, C. Characterization of Lactobacillus coryniformis DSM20001 ${ }^{\top}$ surface protein Cpf mediating coaggregation with and aggregation among pathogens. Appl. Environ. Microbiol. 70, 7078-7085 (2004).

39. Møretrø, T. et al. Sakacin P non-producing Lactobacillus sakeistrains contain homologs of the sakacin P gene clusters. Res. Microbiol. 146, 949-960 (2005).

40. Chung, K.T., Dickson, J.S. \& Crouse, J.D. Attachment and proliferation of bacteria on meat. J. Food Prot. 52, 173-177 (1989).

41. Fleischmann, R.D. et al. Whole genome random sequencing and assembly of Haemophilus influenzae Rd. Science 269, 496-512 (1995).

42. Dudez, A.M. et al. Physical and genetic map of the Lactobacillus sakei $23 \mathrm{~K}$ chromosome. Microbiol. 148, 421-431 (2002).

43. Ewing, B., Hillier, L., Wendl, M.C. \& Green, P. Base-calling of automated sequencer traces using Phred. I accuracy assessment. Genome Res. 8, 175-185 (1998).

44. Gordon, D., Abajian, C. \& Green, P. Consed: a graphical tool for sequence finishing. Genome Res. 8, 198-202 (1998).

45. Hoebeke, M., Nicolas, P. \& Bessières, P. Simultaneous exploration of multiple genomes and computer analysis results. Bioinformatics 19, 859-864 (2003).

46. Chetouani, F., Glaser, P. \& Kunst, F. FindTarget: software for substractive genome analysis. Microbiol. 147, 2643-2649 (2001).

47. Altschul, S.F., Gish, W., Miller, W., Myers, E.W. \& Lipman, D.J. Basic Local Alignment Search Tool. J. Mol. Biol. 215, 403-410 (1990).

48. Saier, M.H., Jr. A functional phylogenetic classification system for transmembrane solute transporters. Microbiol. Mol. Biol. Rev. 64, 354-411 (2000).

49. De Man, J.C., Rogosa, M. \& Sharpe, M.E. A medium for the cultivation of lactobacilli. J. Appl. Bact. 23, 130-135 (1960).

50. Skaugen, M. \& Nes, I.F. Transposition in Lactobacillus sakei: inactivation of a second lactocin S operon by the insertion of IS1520, a new member of the IS3 family of insertion sequences. Microbiol. 146, 1163-1169 (2000). 